\title{
Hurricane Harvey AAR: Preparedness Recommendations Based on Surveillance Data
}

\section{Michelle Carr, Tunde Onafowokan, John Fleming, Tolu Olumuyiwa, Biru Yang, Amanda Eckert}

Informatics, Houston Health Department, Houston, Texas, United States

Objective

To provide recommendations for future preparedness response efforts based on an assessment of the Post-Hurricane Harvey AfterAction Report (AAR).

Introduction

On August 25, 2017 Hurricane Harvey moved onshore near Port Aransas, Texas, eventually overwhelming areas of Houston with between 41-60 inches of rain (Houston Health Department [HHD], 2017). As a category 4 storm, with wind speeds as high as 130 mph, Harvey broke several rainfall records across the state and ended the prolonged period of twelve years in which no major hurricanes had made landfall in the United States (Mersereau, 2017). Harvey ambled at a leisurely pace through Houston and resulted in devastating flooding that destroyed homes and required the evacuation of approximately 37,000 Houstonians to over 78 shelter facilities across the affected area (HHD, 2017). Through concerted efforts, the American Red Cross and the HHD established the shelter at the George R. Brown Convention Center (GRB) and "delivered or coordinated social services, medical and mental health services, disease surveillance and food/sanitary inspection services" for the duration of the need for the shelter (HHD, 2017).

\section{Methods}

Syndromic surveillance data is essential to understanding the health status of affected communities during and after a disaster. For this abstract, we reviewed data collected from different surveillance systems and programs within the Houston Health Department (HHD), namely Real-Time Outbreak and Surveillance (RODS), Houston Electronic Disease Surveillance System (HEDSS) and other program systems, and reports compiled into the AAR. The AAR contained an assessment of the data collected daily during shelter surveillance and helped identify gaps in the implementation of preparedness plans, current procedures, and best practices. HHD's Informatics team was responsible for data collection, training of staff and maintaining a cloud based repository of information on surveillance data and resources. A review of the AAR indicated a need for resources for the general shelter population to address the need for pharmacy data, enhanced behavioral support for individuals with mental health needs, dialysis treatment plans and pharmaceutical needs for patients with respiratory illness or hypertension.

\section{Results}

From August 30, 2017 to September 8, 2017 approximately 3,500 evacuees residing at the GRB shelter were assessed for a variety of medical conditions and complaints. Patient encounters peaked on September 4, 2017, with 705 patient encounters recorded. Data from the AAR suggested there were four most prevalent conditions of immediate need; affecting almost $25 \%$ of the shelter population were hypertension $(10.4 \%)$, mental and behavioral issues $(7.9 \%)$, diabetes $(5.7 \%)$ and dialysis or renal failure $(0.3 \%)$. There were challenges with supply of medications and synchronization of data collection by HHD and partner agencies. The department's Continuity of Operation Plan (COOP) was voluminous and was not easily accessible during the disaster response. The findings from the After- Action Report indicate that disasters present multidimensional health challenges that can overwhelm advance preparations and more needs to be done to address the problems identified from previous disaster responses to improve on future outcomes.

\section{Conclusions}

Syndromic surveillance can be strengthened in the following recommended areas for better incorporation into disaster response plans; pharmacy and health related data and data collection.

The ingestion of pharmacy data by the syndromic surveillance systems could highlight gaps in the supply of needed medications at pharmacies during and post disaster, data from behavioral health clinics could show whether victims of the disaster who suffer mental health issues are able to access care, and whether dialysis treatment plans were continued. Based on the gaps identified, recommendations include integration of pharmacy data into the City's disease surveillance system "ESSENCE" for tracking

ISDS Annual Conference Proceedings 2019. This is an Open Access article distributed under the terms of the Creative Commons AttributionNoncommercial 4.0 Unported License (http://creativecommons.org/licenses/by-nc/3.0/), permitting all non-commercial use, distribution, and reproduction in any medium, provided the original work is properly cited. 
ISDS 2019 Conference Abstracts

prescriptions and OTC purchases, to ensure adequate preparation for disaster stock levels and identification of sources for reordering when stocks run short.

Additionally, it is recommended to revise and standardize data collection tools used during shelter surveillance to streamline the data collection process and to align the data tools of partner agencies, particularly DMAT and Red Cross, to prevent unnecessary duplication of efforts.

Finally, the City's Continuity of Operation Plan (COOP) has been revised since Hurricane Harvey and is periodically assessed and updated. The revised and updated COOP provides a concise and readily accessible document which can be easily reviewed and implemented to support an emergency response.

\section{Acknowledgement}

I would like to thank all of my co-authors for their collaboration on this endeavor.

\section{References}

1. Houston Health Department. (2017). Hurricane Harvey fast facts.

2. Houston Health Department. (2017). After-Action Report/Improvement Plan. Retrieved from

3. Mersereau D. (2017). Hurricane Harvey broke multiple weather records. Mental Floss. Retrieved from http://mentalfloss.com/article/556940/pluto-planet-after- all-new-argument-emerges 